\title{
Cryptococcal Abscess in the Pubic Region and Central Nervous System of an Immunocompetent Patient
}

\author{
Jun Ho Choi (D), Seong Jin Oh (D), Jae Ha Hwang (D), Kwang Seog Kim (D), Sam Yong Lee (D) \\ Department of Plastic and Reconstructive Surgery, Chonnam National University Medical School, Gwangju, Korea
}

\begin{abstract}
Cryptococcus neoformans usually causes an opportunistic infection in immunosuppressed patients. Immunocompetent patients may also develop the infection in the central nervous system or the lungs, but soft tissue infection is uncommon, and the development of a cryptococcal abscess in the pubic region is particularly rare. A 47-year-old male construction worker with headaches and fever visited the hospital due to a soft tissue mass in the pubic region with pus-like discharge. It was detected 6 months previously, and had gradually increased in size, tenderness, and erythema. The granulomatous lesion of the pubic area was excised en bloc, and primary closure was performed. Antifungal agents were used for the systemic infection. Histological examination identified the specimen as a fungal infection suspected to be cryptococcosis. During the 3-month follow-up period, there was no recurrence, infections in other areas, or major wound complications. This case is an example of a cryptococcal abscess with dissemination in the pubic region of an immunocompetent patient. There is no established cure for subcutaneous cryptococcal abscesses, but surgical excision, which can simultaneously achieve accurate diagnosis and treatment, is considered to be an effective treatment method.
\end{abstract}

Keywords: Abscess; Cryptococcus; Immunocompetence

\section{Introduction}

Cryptococcus neoformans is an encapsulated fungal organism that is known to cause opportunistic infections in immunocompromised hosts such as human immunodeficiency virus (HIV)-positive patients, diabetics, and patients using immunosuppressants [1]. Cryptococcus mainly infects the lungs initially [2] and disseminates through the blood into the meninges and other organs. Infection in immunocompromised hosts can cause systemic infection and can be fatal; in particular, mortality due to cryptococcal meningitis can reach $30 \%$ to $50 \%$ [3]. Although uncommon, C. neoformans can also cause infections in the skin, bone, and soft tissue [4]. In this paper, we report a rare case of disseminated cryptococcosis in the meninges and soft tissue of the pubic region, the first to be reported in an immunocompetent host. The patient provided informed consent for the publication of his clinical and radiological data. The study was approved by the Institutional Review Board of Chonnam National University Hospital (IRB No. CNUH-2020-194) and performed in accordance with the principles of the Declaration of Helsinki.

\section{Case}

A 47-year-old male construction worker with headaches and fever visited the hospital

\section{Case Report}

Received: January 13, 2021

Revised: January 25, 2021

Accepted: January 27, 2021

Corresponding author:

Jae Ha Hwang M.D., Ph.D.

Department of Plastic and Reconstructive Surgery, Chonnam National University Medical School, 42 Jebong-ro, Dong-gu, Gwangju 61469, Korea

Tel: +82-62-220-6368

Fax: +82-62-227-1639

E-mail: psjhhwang@daum.net

This is an Open Access article distributed under the terms of the Creative Commons Attribution Non-Commercial License (https://creativecommons.org/licenses/by-nc/4.0/) which permits unrestricted non-commercial use, distribution, and reproduction in any medium, provided the original work is properly cited.

C) 2021 Korean Wound Management Society 

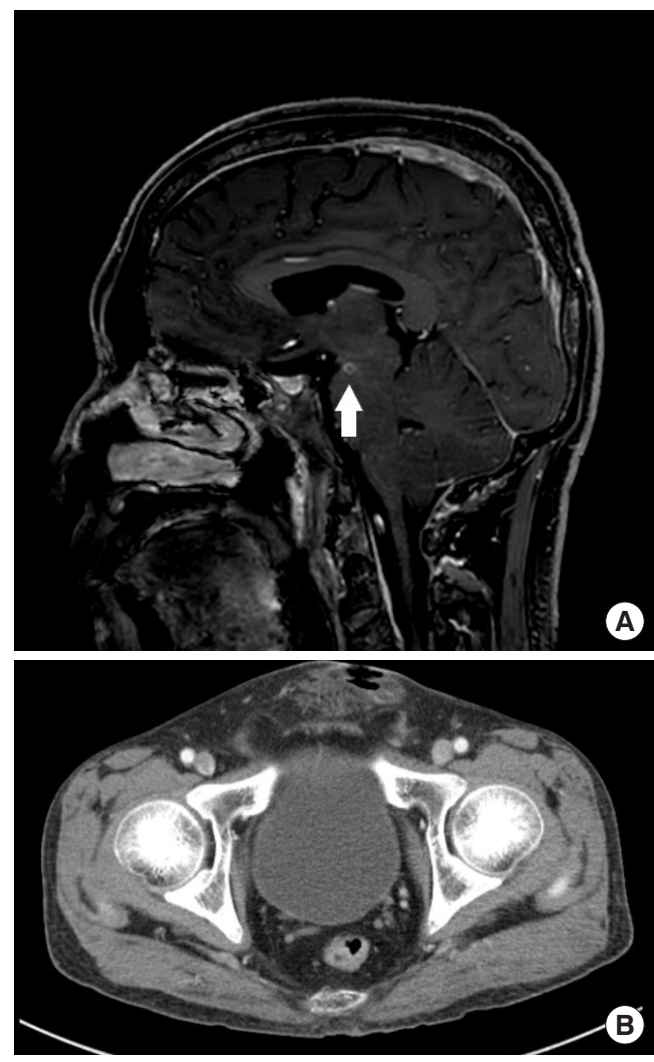

Fig. 1. Preoperative imaging study. The imaging study findings show the dissemination of cryptococcosis to the meninges and pubic region. (A) Brain magnetic resonance imaging revealed evidence of abscess in the left cerebral peduncle (white arrow). (B) Abdominal-pelvis computed tomography showed defects in the anterior pubic wall with soft tissue stranding in the subcutaneous fat layer.

due to a soft tissue mass in the pubic region with pus-like discharge. It was in the location of an infected wound detected 6 months previously, which had gradually increased in size, tenderness, and erythema. An excisional biopsy was performed at a local clinic a month prior to the current presentation, and the biopsy showed an abscess with fat necrosis, as well as wound dehiscence. The patient had been treated with medication for atypical pneumonia by nontuberculous mycobacterium 6 months earlier. He had controlled diabetes mellitus and had received a penile foreign body injection in the pubic region 3 years ago. He had a bitemporal pulsatile headache and fever. The patient was examined by a neurologist. Brain magnetic resonance imaging revealed evidence of meningitis and abscesses in the left cerebral peduncle, cerebellar folia and right basal ganglia (Fig. 1A). Cerebrospinal fluid (CSF) culture confirmed C. neoformans. Abdominal-pelvis computed tomography showed defects in the anterior pubic wall with soft

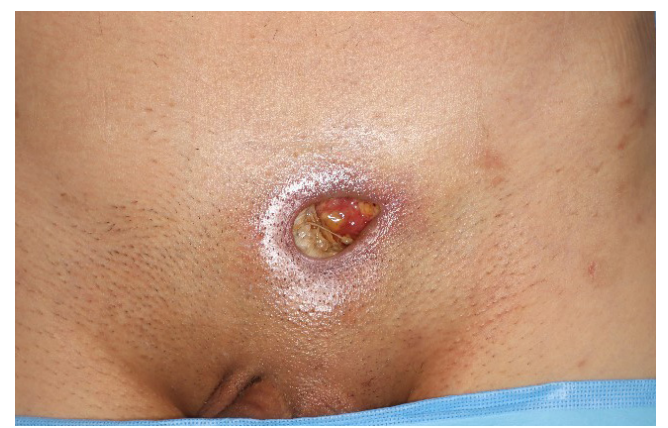

Fig. 2. Preoperative photograph of the pubic cryptococcal abscess. The granulomatous lesion of the pubic area was excised in en bloc, and primary closure was performed.

tissue stranding in the subcutaneous fat layer (Fig. 1B). In laboratory findings, inflammatory markers were within normal range, but CSF white blood cell (WBC) counts and CSF protein levels were elevated (WBC: 7,100/ $\mu \mathrm{L}, \mathrm{C}$-reactive protein: $0.20 \mathrm{mg} / \mathrm{dL}, \mathrm{CSF}$ WBC counts: $184 / \mu \mathrm{L}$ [neutrophil 45\%, lymphocyte 41\%], CSF protein levels: $143.9 \mathrm{mg} / \mathrm{dL}$ ).

An en bloc excision was planned. During en bloc excision, we confirmed that the abscess had spread to the penile root and suspensory ligament, and through careful dissection, we completed the operation without major injury (Fig. 2).

There were no postoperative complications such as erectile dysfunction reported. The biopsy result confirmed necrotizing granulomatous inflammation and fat necrosis accompanied by a fungal infection suspected to be cryptococcosis, which was subsequently confirmed by periodic acid-Schiff staining and methenamine silver staining (Fig. 3).

The patient received intravenous amphotericin B liposomal (AmBisome) $4 \mathrm{mg} / \mathrm{kg} / \mathrm{day}$ and oral fluconazole $800 \mathrm{mg} / \mathrm{day}$ for 2 weeks after consultation with the neurologist, followed by 8 weeks of consolidation treatment with oral fluconazole $800 \mathrm{mg} /$ day. During the 3-month follow-up period, there was no recurrence, infections in other areas, or major wound complications (Fig. 4).

\section{Discussion}

Cryptococcus is a fungus and an encapsulated yeast, and C. neoformans and Cryptococcus gattii are known to cause infections in humans. Cryptococci can cause cryptococcosis in both immunocompetent and immunocompromised patients, but cryptococci primarily cause opportunistic infections in immunocompromised patients such as patients who are HIV-positive, diabetic, on immunosuppressants, have chronic kidney 

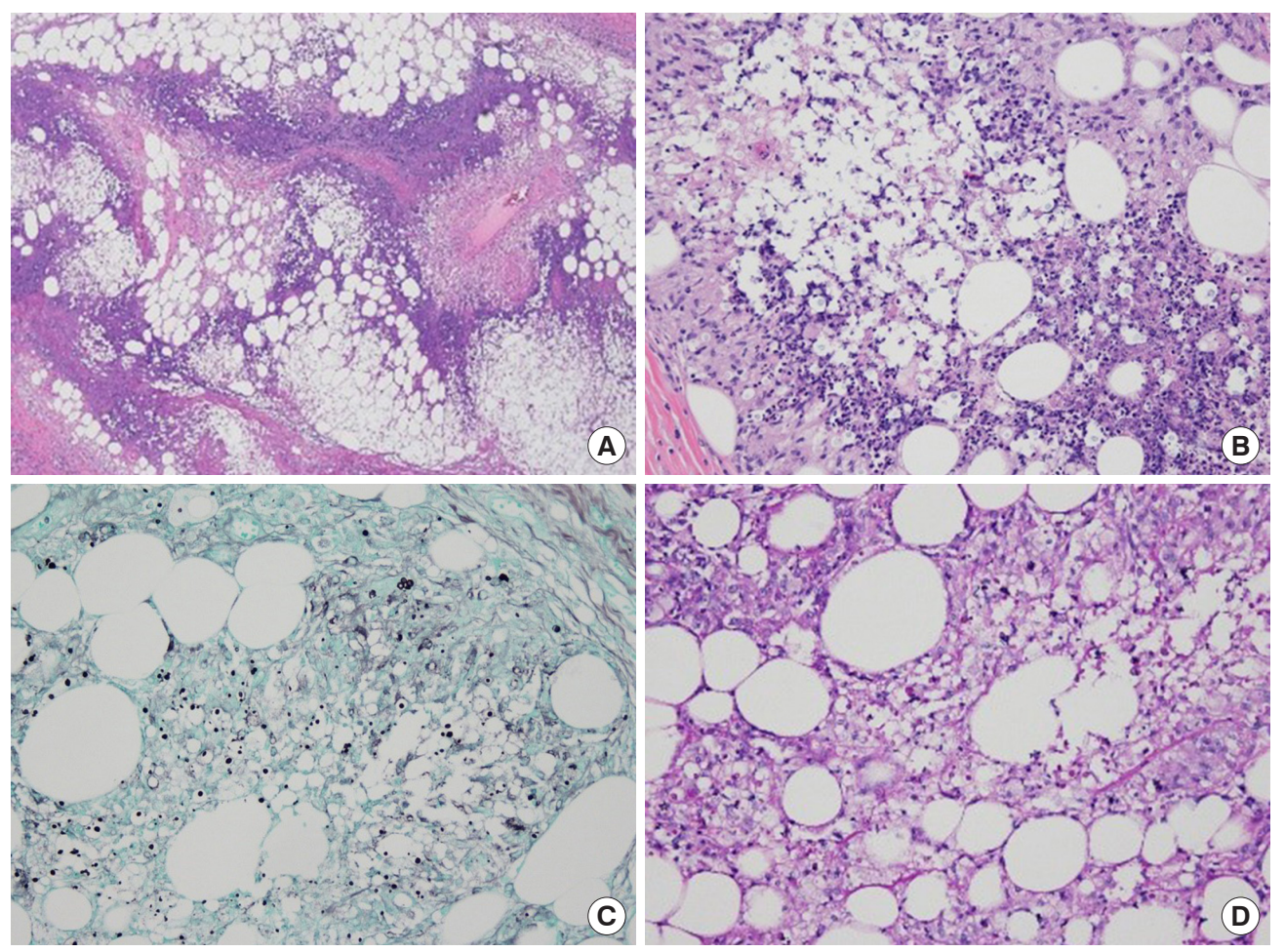

Fig. 3. Histopathologic findings. The pathologic result confirmed that the extensive necrotizing granulomatous inflammation and fat necrosis were associated with fungal infection, which was apparently cryptococcosis. Special stains were administered on the fungal capsules, and the cryptococcal infection was confirmed by wound culture. (A) H\&E stain, $\times 40$. (B) H\&E stain, × 100. (C) Methenamine silver stain, $\times 100$. (D) Periodic acid-Schiff stain, $\times 100$.
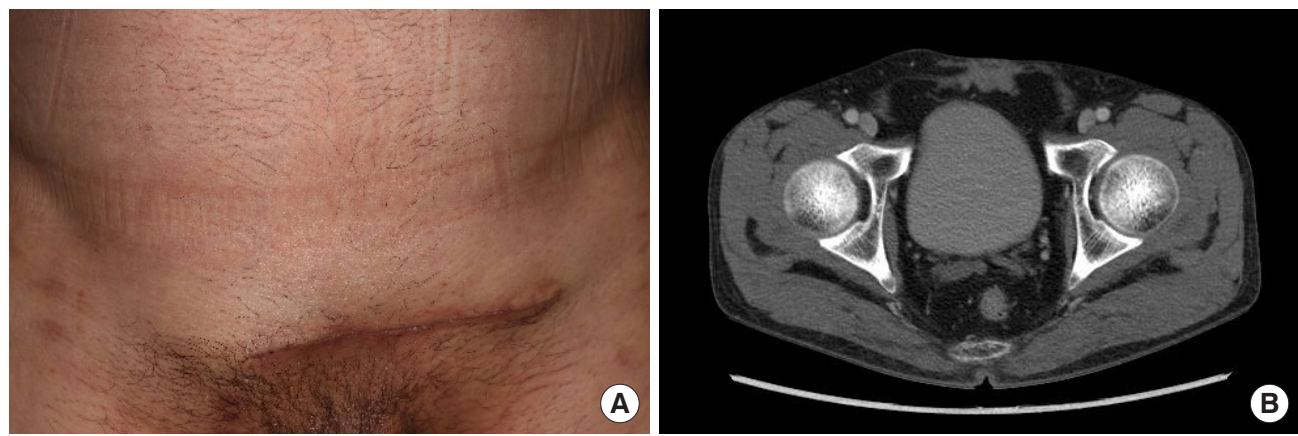

Fig. 4. Follow-up photograph and imaging study. (A) A follow-up photo after 1 month and (B) follow-up imaging study after 3 months. Although the headache persisted, there were no recurred soft tissue infections or major wound complications.

disease or liver cirrhosis, or are pregnant. C. neoformans usually causes infections in the lung first, after which it can then spread through the circulation and cause meningitis as well as central nervous system abscesses, and even lead to fatal cryptococcal disease. Extrapulmonary and extracranial cases are rare, but infections may occur in the skin, bone, and soft tissue.

In a recent study [5,6], seven cases of extrapulmonary and extracranial cryptococcosis in immunocompetent patients were reported, with the infections located in the perivertebral region, thigh, sternum, and neck; there were no reported cases in the pubic region. Our patient developed a subcutaneous abscess in the pubic region, and the infection was granulomatous in nature. It was accompanied by a cranial infection at the time of admission. According to a previous study [5], most cutaneous and subcutaneous lesions indicate disseminated cryptococcal infections, and are often accompanied by pulmonary or cranial infections. Thus, a study reported that soft tissue infection can be used as a key marker for cryptococcal dissemina- 
tion [7]. Therefore, when soft tissue infection is detected, screening for lung and brain dissemination is recommended.

The features of soft tissue cryptococcal infection include abscess, vesicle, purpura, nodule, ulcer, granuloma, pustule, and cellulitis [8]. In pulmonary cryptococcosis, clinical findings such as cough, sputum, and chest pain are observed, and it is often preceded by malignancy or tuberculosis. There are reports that after the invasion of the central nervous system, the mortality in immunosuppressed patients reaches $30 \%$ to $50 \%$; although symptoms of meningitis such as headache, fever, and visual loss can be observed, there may not be any typical symptoms present. In addition, arthritis, hepatitis, thyroiditis, sinusitis, and lymphadenopathy can be reported. Since most symptoms are not specific, they are often attributed to other diseases, and tissue culture or biopsy is necessary to confirm the diagnosis. However, due to the low fungal burden in the early stages of infection, and the fact that fungemia rarely occurs in immunocompetent patients, results may be negative even with repeated cultures. Therefore, obtaining tissue through surgical intervention is recommended for a more accurate diagnosis. Cryptococcus capsules are made up of glucuronoxylomannan and galactoxylomannan polysaccharides, so pathologic results are more reliable with special stains such as the Gomori methenamine silver stain and the mucicarmine stain. Our case was confirmed pathologically via invasive excisional biopsy and methenamine silver stain.

This patient was immunocompetent, but because he worked in the construction industry and was in an environment exposed to contaminated dust, and because he had a history of nontuberculous mycobacterial pneumonia, he may have been vulnerable to pulmonary infections and even could have been misdiagnosed. In addition, the patient underwent penile foreign body injection through the pubic region in the past, which may have resulted in scar formation or foreign body reaction in the area; this could have led to local immunosuppression, providing the ideal conditions for subcutaneous dissemination that could have promoted cryptococcal dissemination.

This case is an example of a cryptococcal abscess with dissemination in the pubic region of an immunocompetent patient. We were able to accurately diagnose and treat the patient via surgical excision and antifungal agents. While there is no established cure for subcutaneous cryptococcal abscesses, surgical excision, which can simultaneously achieve accurate diagnosis and treatment, is considered to be an effective treatment method.

\section{Conflict of interest}

Jae Ha Hwang is an editorial board member of the journal but was not involved in the peer reviewer selection, evaluation, or decision process of this article. No other potential conflicts of interest relevant to this article were reported.

\section{ORCID iDs}

Jun Ho Choi

Seong Jin $\mathrm{Oh}$

Jae Ha Hwang

Kwang Seog Kim

Sam Yong Lee

https://orcid.org/0000-0002-4848-517X https://orcid.org/0000-0003-4057-9398 https://orcid.org/0000-0001-6992-8067 https://orcid.org/0000-0002-6766-4640 https://orcid.org/0000-0002-3185-2519

\section{References}

1. Chayakulkeeree M, Perfect JR. Cryptococcosis. Infect Dis Clin North Am 2006;20:507-44.

2. Setianingrum F, Rautemaa-Richardson R, Denning DW, et al. Pulmonary cryptococcosis: a review of pathobiology and clinical aspects. Med Mycol 2019;57:133-50.

3. Abassi M, Boulware DR, Rhein J. Cryptococcal meningitis: diagnosis and management update. Curr Trop Med Rep 2015;2:90-9.

4. Al-Tawfiq JA, Ghandour J. Cryptococcus neoformans abscess and osteomyelitis in an immunocompetent patient with tuberculous lymphadenitis. Infection 2007;35:377-82.

5. Ruan Q, Zhu Y, Chen S, et al. Disseminated cryptococcosis with recurrent multiple abscesses in an immunocompetent patient: a case report and literature review. BMC Infect Dis 2017;17:369.

6. Park SW, Choi JY, Kim AY, et al. A case of cryptococcal abscess involving deep neck space in an immunocompetent patient. Korean J Otorhinolaryngol-Head Neck Surg 2011; 54:638-41.

7. Srivastava GN, Tilak R, Yadav J, et al. Cutaneous cryptococcus: marker for disseminated infection. BMJ Case Rep 2015;2015:bcr2015210898.

8. Wang J, Bartelt L, Yu D, et al. Primary cutaneous cryptococcosis treated with debridement and fluconazole monotherapy in an immunosuppressed patient: a case report and review of the literature. Case Rep Infect Dis 2015;2015: 131356. 